\title{
Towards a Systematic Review of Automated Feedback Generation for Programming Exercises
}

\author{
Hieke Keuning \\ Open University of the \\ Netherlands and Windesheim \\ University of Applied Sciences \\ hw.keuning@windesheim.nl
}

\author{
Johan Jeuring \\ Utrecht University and Open \\ University of the Netherlands \\ j.t.jeuring@uu.nl
}

\author{
Bastiaan Heeren \\ Open University of the \\ Netherlands \\ bastiaan.heeren@ou.nl
}

\begin{abstract}
Formative feedback, aimed at helping students to improve their work, is an important factor in learning. Many tools that offer programming exercises provide automated feedback on student solutions. We are performing a systematic literature review to find out what kind of feedback is provided, which techniques are used to generate the feedback, how adaptable the feedback is, and how these tools are evaluated. We have designed a labelling to classify the tools, and use Narciss' feedback content categories to classify feedback messages. We report on the results of the first iteration of our search in which we coded 69 tools. We have found that tools do not often give feedback on fixing problems and taking a next step, and that teachers cannot easily adapt tools to their own needs.
\end{abstract}

\section{Keywords}

systematic literature review; automated feedback; programming tools; learning programming

\section{INTRODUCTION}

Tools that support students in learning programming have been developed since the 1960s [6]. Such tools provide a simplified development environment, use visualisation or animation to give better insight in running a program, guide students towards a correct program by means of hints and feedback messages, or automatically grade student solutions [11].

Two important reasons to develop tools that support learning programming are: (1) learning programming is hard, and students need help to make progress, and (2) programming courses are taken by many thousands of students all over the world, and helping students individually with their problems requires a huge time investment of teachers.

Feedback is an important factor in learning [22]. Boud and Molloy define feedback as 'the process whereby learners obtain information about their work in order to appreciate the similarities and differences between the appropriate standards for any given work, and the qualities of the

Permission to make digital or hard copies of part or all of this work for personal or classroom use is granted without fee provided that copies are not made or distributed for profit or commercial advantage and that copies bear this notice and the full citation on the first page. Copyrights for third-party components of this work must be honored.

ITiCSE '16, July 11-13, 2016, Arequipa, Peru.

(C) 2016 Copyright held by the owner/author(s).

ACM ISBN 978-1-4503-4231-5/16/07.

DOI: http://dx.doi.org/10.1145/2899415.2899422

\section{(c) (i)}

This work is licensed under a Creative Commons Attribution

International 4.0 License. work itself, in order to generate improved work' [2]. Thus defined, feedback is formative: it consists of 'information communicated to the learner with the intention to modify his or her thinking or behavior for the purpose of improving learning' [22]. Summative feedback in the form of grades or percentages for assessments also provides some information about the work of a learner. However, the information a grade gives about similarities and differences between the appropriate standards for any given work, and the qualities of the learner's work, is usually only superficial. In this paper we focus on the formative kind of feedback as defined above. Formative feedback comes in many variants, and the kind of formative feedback together with student characteristics greatly influences the effect of feedback [15].

Given the role of feedback in learning, we want to find out what kind of feedback is provided by tools that support learning programming. What is the nature of the feedback, how is it generated, can a teacher adapt it, and what can we say about its quality and effect? To answer these questions, we are performing a systematic literature review of automated feedback generation for programming exercises.

A systematic literature review (SLR) is a research method that identifies and investigates all relevant publications on a particular topic. A research plan is designed in advance, and the execution of this plan is documented in detail, allowing insight into the rigorousness of the research.

This paper reports on the results found in the first iteration of our search for relevant papers. ${ }^{1}$ We searched for related overviews on tools for learning programming and executed the first step of 'backward snowballing' by selecting relevant references from the papers we found. Our search until now has resulted in a set of 102 papers, describing 69 different tools. We have designed a labelling to classify the tools and provide answers to our research questions. Although not yet complete, this collection is representative and large enough to report some findings.

This review makes the following contributions:

- We analyse what kind of feedback is used in tools that support a student in learning programming. Although quite a few reviews analyse such tools, none of them looks at the feedback provided by these tools.

- We relate the feedback content to its technology, and to the adaptability of the tool and the feedback.

Section 2 discusses related reviews of tools for learning

\footnotetext{
${ }^{1}$ This is a short version of our work. We have published a Technical Report containing detailed information and the complete reference list [12].
} 
programming. Section 3 gives our research questions and research method. Section 4 shows and discusses the results. Section 5 concludes the paper.

\section{RELATED WORK}

We have found almost twenty reviews of tools for learning programming, mostly on automated assessment (AA) tools $[1,3,6,10,20,21,23]$ or learning environments for programming $[4,5,7,9,11,13,14,18,19,24]$. Generating feedback is important for both kinds of tools. Most AA tools only grade student solutions, but some tools also provide elaborated feedback, and can be used to support learning [1].

Most reviews describe the features and characteristics of a number of tools, identify challenges, and direct future research. Except for Ihantola et al. [10] and the review in progress by Nesbit et al. [17], authors select papers and tools using unknown criteria, some mention qualitative factors such as impact (citations) or the thoroughness of the evaluation of the tool. Most studies do not strive for completeness. The scope of the tools that are described varies greatly. Tools are usually categorised, but there is no agreement on the naming of the different categories. Very few papers discuss technical aspects.

Our review distinguishes itself from the above reviews by focusing on the aspect of generating feedback in learning tools for programming. Furthermore, we employ a more systematic approach than almost all of the above papers.

\section{METHOD}

Performing an SLR requires an in depth description of the research method. This section describes our research questions, the criteria and the search process.

\subsection{Research questions}

The following four research questions guide our review:

RQ1. What is the nature of the feedback that is generated?

RQ2. Which techniques are used to generate the feedback?

RQ3. How can the tool be adapted by teachers, to create exercises and to influence the feedback?

RQ4. What is known about the quality and effectiveness of the feedback or tool?

\subsection{Criteria}

We have defined a set of inclusion and exclusion criteria that direct our research and target the characteristics of the papers and the tools described therein.

General. We include journal and conference papers in English, and Master or PhD theses, or technical reports only if a journal or conference paper is available on the same topic. The publication describes a tool of which at least a prototype has been constructed. We exclude papers shorter than four pages.

Functionality. Developing a program that solves a particular problem is an important learning objective for learning programming. We include tools in which students work on programming exercises of class 2 or higher from the classification of Le and Pinkwart [13]. Class 1 exercises have a single solution, and are often quiz-like questions. Class 2 exercises can be solved by different implementation variants. Usually a program skeleton or other information about the solution strategy is provided, but variations in the implementation are allowed. Finally, class 3 exercises can be solved by applying alternative solution strategies, which we interpret as allowing different algorithms as well as different steps to arrive at a solution. The type of exercises that a learning tool supports determines to a large extent how difficult it is to generate feedback. Tools provide automated, textual feedback on (partial) solutions, targeted at the student. We exclude tools that only produce a final grade.

Domain. We include tools that support a high-level, general purpose, textual programming language used in the industry and/or taught at universities, including pseudocode. We exclude visual programming tools (block programming, flowcharts), and tools that focus on one aspect of programming, such as recursion or multi-threading.

We select papers and tools that satisfy all inclusion criteria and none of the exclusion criteria. We have included some theses because they have been cited often.

\subsection{Search process}

The starting point of our search for papers was the collection of 17 review papers given in Section 2. Two authors of this SLR independently selected relevant references from these reviews. Then two authors independently looked at the full text of the papers in the union of these selections, to exclude papers not meeting the criteria. After discussing the differences, we assembled a final list of papers. We had to exclude a small number of papers that we could not find after an extensive search and, in some cases, contacting the authors. Some excluded papers point to a potentially interesting tool. We checked if these papers mention a better reference that we could add to our selection.

Often multiple papers have been written on (versions of) a single tool. We searched for all publications on a tool by looking at references from and to papers already found, and searching for other relevant publications by the authors. We selected the most recent and complete papers about a tool.

Starting with an initial selection of 197 papers, we ended up with a total of 102 papers describing 69 different tools.

\section{RESULTS AND DISCUSSION}

To systematically encode the information in the papers, we use a labelling based on the answers to the research questions we expected to get, refined by encoding a small set of randomly selected papers. One of the authors encoded the complete set of papers. Whenever there were questions about the coding of a paper, another author checked. In total, $28 \%$ of the codings were handled by two authors. A third author joined the general discussions about the coding. When necessary, we made adjustments to the labelling.

The results we have found so far in our SLR are shown in the table, sorted by exercise class. We record the highest exercise class a tool supports. A majority of the tools supports imperative languages, including object-oriented languages (Java, $\mathrm{C}$ and $\mathrm{C}++$, and earlier FORTRAN, Ada). Fewer tools support a functional language (Lisp, Haskell) or logic programming (Prolog). Three recent tools focus on web scripting languages (PHP, JavaScript). The remaining tools support multiple languages, and are often test-based AA systems. Tools often only support a subset of the features of a programming language. 
The remainder of this section describes the labelling for the research questions, provides examples and concludes with some observations. We refer to the tools in the table by their name in a SMALL CAPS font, or the first author and year of the most recent paper (AUTHOR00) on the tool we have used.

\subsection{RQ1 Feedback type}

Narciss [16] describes a 'content-related classification of feedback components' for computer-based learning environments, in which the categories target different aspects of the instructional context, such as task rules, errors and procedural knowledge. We use these categories and extend them with representative subcategories identified in the selected papers. Narciss also considers the functions and presentation of feedback, which are related to the effectiveness of tutoring. We do not include these aspects in our review because it is often unclear how a tool or technique is used in practice (e.g. formative as well as summative).

Narciss first gives simple feedback components: 'Knowledge of performance for a set of tasks', 'Knowledge of result/response' and 'Knowledge of the correct results'. These types of feedback are not intended to 'generate improved work', a requirement in the feedback definition by Boud and Molloy. Because we focus on formative feedback on a single exercise, we do not identify these types in our coding.

The next five types are elaborated feedback components. Each type addresses an element of the instructional context. We provide several examples to illustrate how the different types are instantiated in learning tools for programming. We have also identified a few examples of feedback messages that do not fit into our classification, such as motivating feedback.

\section{Knowledge about task constraints (KTC)}

- Hints on task requirements (TR). A task requirement for a programming exercise can be to use a particular language construct or to not use a particular library method. An example can be found in the BASIC InstructionaL Program (BIP): 'Wait. Something is missing. For this task, your program should also include the following basic statement $(s)$ : FOR'

- Hints on task-processing rules (TPR). These hints provide general information on how to approach the exercise and do not consider the current work of a student.

\section{Knowledge about concepts (KC)}

- Explanations on subject matter (EXP), generated while a student is working on an exercise. The AsK-Elle programming tutor refers to relevant internet sources when a student encounters certain language constructs.

- Examples illustrating concepts (EXA). THE LISP TUTOR uses examples in its tutoring dialogue. For example, after a student has made a mistake, the tutor responds with: 'Since you seem to be having trouble with the recursive cases, let us work through some examples and figure out the conditions and actions for each of these cases.'

\section{Knowledge about mistakes (KM)}

KM feedback messages have a type and a level of detail. A level of detail can be basic $(\bigcirc)$, which can be a numerical value (total number of mistakes, grade, percentage), a location (line number code fragment), or a short type identifier such as 'compiler error'; or detailed ( ), which is a de- scription of the mistake, possibly combined with some basic elements. We distinguish five different mistake types.

- Test failures (TF). A failed test indicates that a program does not produce the expected output. Coffman10, an AA tool for web programming, provides detailed feedback on test results, showing the informative name of the test cases (such as 'checkInvalidLogin'), a colour indicating their success and the reason why a particular test case failed. We found this type of feedback, which resembles the output of professional testing tools, in many AA tools.

- Compiler errors (CE) are syntactic errors (incorrect spelling, missing brackets) or semantic errors (type mismatches, unknown variables) that can be detected by a compiler and are not specific for an exercise. Feedback on compiler errors might be the output of a compiler that is passed on to the student. Test-based AA systems often provide compiler output as feedback, because successful compilation is a prerequisite for executing tests.

- Solution errors (SE) can be found in programs that do not show the behaviour that a particular exercise requires, and can be runtime errors (the program crashes) or logic errors (the program does not do what is required), or the program uses an alternative algorithm that is not accepted. AutoLEP describes the results of matching the student program with several model programs, comparing aspects such as size, structure, and statements.

- Style issues (SI), such as untidy formatting, inconsistent naming or lack of comments, are not serious mistakes that affect the behaviour of a program. However, many teachers consider learning a good programming style important for novice programmers. The feedback generated by the tool of JACKSON00 shows a list of style metrics such as ' $4.0 \%$ comment lines' and ' $20.9 \%$ indentation'.

- Performance issues (PI). A student program takes too long to run or uses more resources than required. NAUR64, one of the earliest systems, checks one particular exercise that lets students write an algorithm for finding the root of a given function. The system gives performance feedback for each test case, such as 'No convergence after 100 calls.'

\section{Knowledge about how to proceed (KH)}

- Bug-related hints for error correction (EC). Sometimes it is difficult to see the difference between KM feedback and EC. We identify feedback as EC if the feedback clearly focuses on what the student should do to correct a mistake. JITS gives feedback on typing errors, such as 'Would you like to replace smu with sum?'

- Task-processing steps (TPS). A TPS hint contains information about the next step a student has to take to come closer to a solution. The Prolog tutor (Hong04) provides a guided programming phase. If a student asks for help in this phase, the tutor will respond with a hint on how to proceed and generates a template for the student to fill out: 'You can use a programming technique that processes a list until it is empty by splitting it into the head and the tail, making a recursive call with the tail.'

Each of these types of feedback has a level of detail: a hint (D) that may be a in the form of a suggestion, a question, or an example; a solution ( $)$ that directly shows what needs to be done to correct an error or to execute the next step; or both hints and solutions $(\mathbf{)})$. 


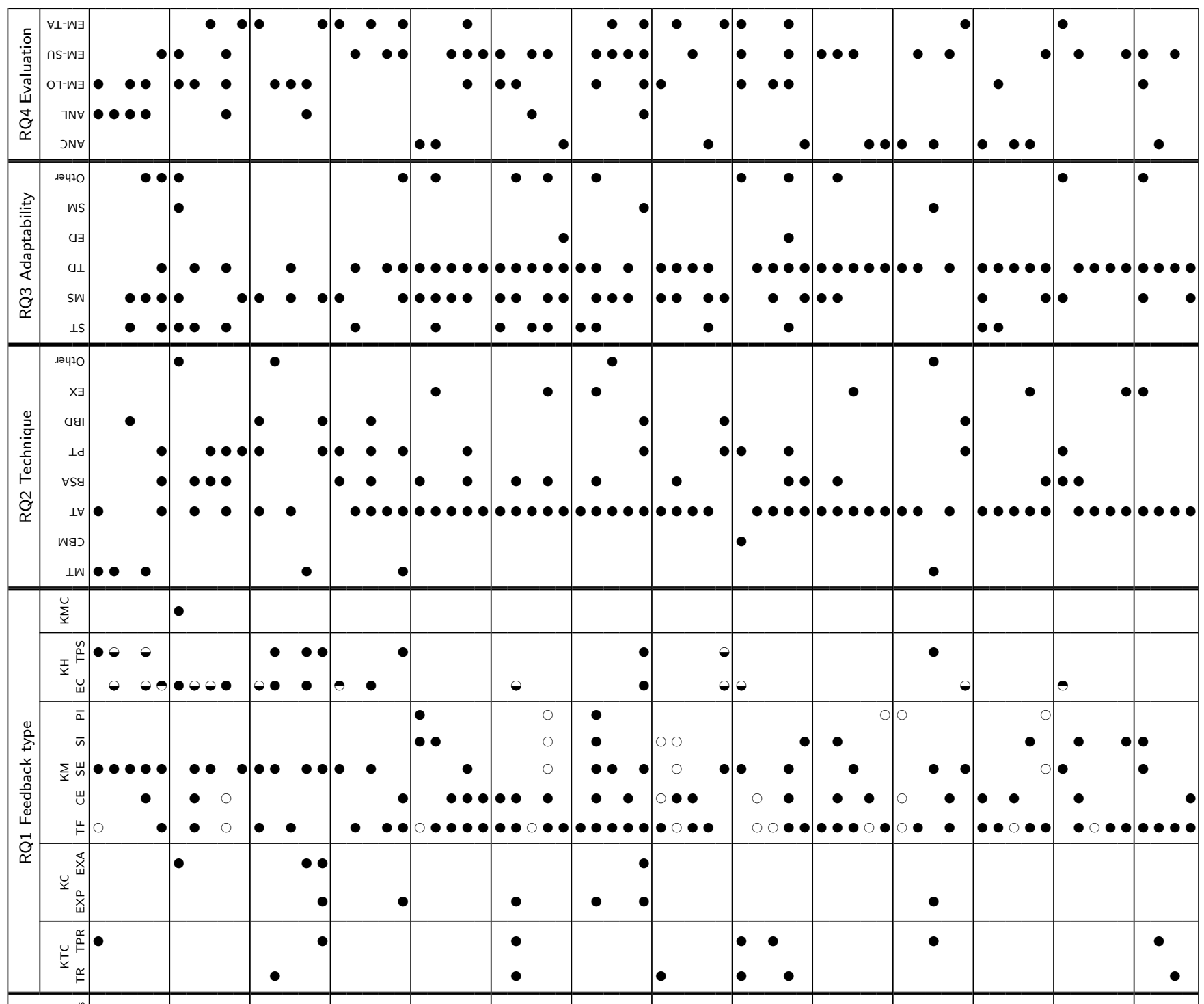

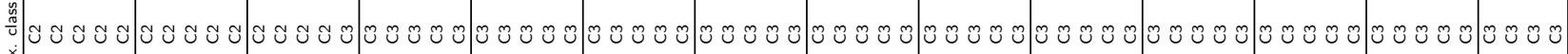

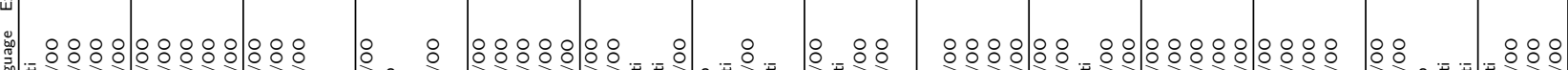

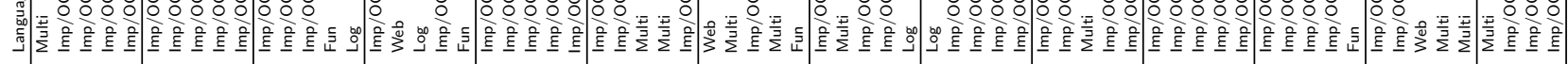

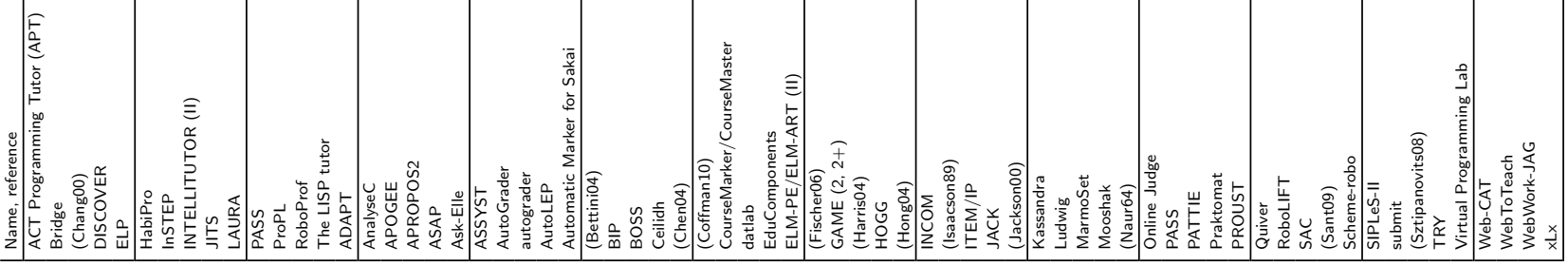




\section{Knowledge about meta-cognition (KMC)}

We have only found one example of KMC so far. HABIPRO provides a 'simulated student' that responds to a solution by checking if a student really knows why an answer is correct.

\subsection{RQ2 Technique}

\section{General ITS techniques}

- Tools that use model tracing (MT) generate feedback on the process that the student is following. Student steps are compared to production rules and buggy rules.

- Constraint-based modelling (CBM). A constraint-based tool checks a student program against predefined solution constraints, such as the presence of a for-loop or the calling of a method with certain parameters, and generates error messages for violated constraints.

\section{Domain-specific techniques for programming}

- Dynamic code analysis using automated testing (AT). The most basic form of AT is running a program and comparing the output to the expected output. More advanced techniques are unit testing and property-based testing, often implemented using existing test frameworks (e.g. JUnit). Most tools that use automated testing support C3 exercises, because black-box testing does not require using a specific algorithm or design process.

- Basic static analysis (BSA) analyses a program (source code or byte code) without running it, and can be used to detect misunderstood concepts, the absence or presence of certain code structures, and to give hints on fixing these mistakes [23]. Some tools use BSA for calculating metrics, such as cyclomatic complexity or number of comments.

- Program transformations (PT) transform a program into another program in the same language (e.g. normalisation) or a different language (e.g. to bytecode). Transformations are often used together with static code analysis to match a student program with a model program.

- Intention-based diagnosis (IBD) uses a knowledge base of programming goals, plans or (buggy) rules to match with a student program to find out which strategy the student uses to solve an exercise.

- External tools (EX) other than testing tools, for example CheckStyle for checking code conventions and FindBugs for finding bugs, and standard compilers. These tools are not the work of the authors themselves and papers do not usually elaborate on the inner workings of the external tools used. If a tool uses automated testing, for which compilation is a prerequisite, we do not use this label.

\section{Other techniques}

Tools use various A.I. techniques, such as natural language processing (PROPL) or machine learning (DATLAB). We expect that some of the techniques in this category will develop into their own category. For example, we have noticed the use of data analysis in quite a number of recent publications: large data sets with student solutions to exercises are used to generate feedback.

\subsection{RQ3 Adaptability}

Which input to a tool can be adapted by teachers without recompiling the tool? Using such input a teacher constructs a new exercise or influences the generated feedback, without too much effort or specialised knowledge.
- Solution templates (ST) (e.g. skeleton programs and projects) presented to students for didactic or practical purposes.

- Model solutions (MS): correct solutions to a programming exercise.

- Test data (TD), such as expected output and test cases.

- Error data (ED), such as bug libraries, buggy solutions, buggy rules and correction rules. Error data usually specify common mistakes for an exercise, and may include corresponding solutions.

- Other. Some tools let a teacher define feedback messages (ASK-ELLE), or configure how much feedback should be given (COURSEMARKER).

Another aspect we consider is the adaptability of the feedback generation based on a student model (SM). A student model contains information on the capabilities and level of the student, and may be used to personalise the feedback.

\subsection{RQ4 Quality}

Tools have been evaluated using a large variety of methods. We use the three main types for the assessment of tools distinguished by Gross and Powers [8].

- Anecdotal (ANC) assessment is based on the experiences and observations of researchers or teachers with using the tool. We will not attach this label if another type has been applied as well, because we consider anecdotal assessment to be inferior to the other types.

- Analytical (ANL) assessment compares the characteristics of a tool to a set of criteria related to usability or a learning theory. For example, The LISP TUTOR and JITS are based on ACT-R.

- Empirical assessment analyses qualitative or quantitative data. We distinguish three types of empirical assessment: looking at the learning outcome (EM-LO) (mistakes, grades, pass rates) after students have used the tool, and observing tool use; student and teacher surveys (EM-SU) and interviews on experiences with the tool; and technical analysis (EM-TA) to verify whether a tool can correctly recognise (in)correct solutions and generate appropriate hints. Tool output for a set of student submissions is compared to an analysis by a human tutor.

\subsection{Discussion}

Feedback about mistakes is the most common type of feedback, with information on test failures as the largest subcategory. Generating feedback based on tests is a useful way to point out errors, emphasizes the importance of testing, and is relatively easy to implement. We have found feedback on solution errors in fewer tools, and with varying depth and detail. Most tools that support C3 exercises give no 'knowledge on how to proceed (KH)' feedback. According to Boud and Molloy's definition, these tools lack the means to really help a student. In general, the feedback that tools generate is not that diverse, and mainly focused on identifying mistakes. Exceptions are tools that only offer C2 exercises. These tools more often provide KH feedback. However, these tools do not support alternative solution strategies, and may restrict students in their problem solving process.

We have found that tools use various dynamic and static analysis techniques. More sophisticated techniques, such as model tracing and intention-based diagnosis, appear to complicate adding new exercises and adjusting the tool. 
Whether or not a tool can be adapted easily depends on the amount and complexity of the input. Very few papers explicitly describe this, or even address the role of the teacher, so we assume that the tool can only be adjusted by developers. When a publication does describe how an exercise can be added, it is sometimes not clear how difficult this is. We conclude that teachers cannot easily adapt tools to their own needs, except for test-based AA systems.

Most tools provide some form of evaluation, although for $23 \%$ of the tools we could only find anecdotal evidence, or none at all. The evaluation of a tool may not be directly related to the quality of the feedback, so the results only give a general idea of how much attention was spent on evaluation. The many different evaluation methods make it difficult to assess the effectiveness of the feedback. Moreover, the quality (e.g. the presence of control groups, pre- and post-tests, group size) of empirical assessment varies greatly. Finally, the description of the evaluation method and results often lacks clarity and detail.

\section{CONCLUSIONS AND FUTURE WORK}

We have analysed and categorised the feedback generation in 69 tools for learning programming, selected from 17 earlier reviews. Although our search is not yet complete, our take-home message is that tools for learning programming should offer more diverse feedback, and better support teachers in specifying exercises. To complete this SLR, we continue our 'backward snowballing' approach by searching the papers we have found so far for relevant references, and we will conduct a search on multiple databases. Lastly, we will further analyse the results by relating them to programming concepts that students find difficult, common novice programming errors, and human tutoring strategies.

\section{Acknowledgements}

This research is supported by the Netherlands Organisation for Scientific Research (NWO), grant number 023.005.063.

\section{REFERENCES}

[1] K. M. Ala-Mutka. A survey of automated assessment approaches for programming assignments. Computer Science Education, 15(2):83-102, 2005.

[2] D. Boud and E. Molloy, editors. Feedback in higher and professional education: understanding it and doing it well. 2012.

[3] J. C. Caiza and J. M. Del Alamo. Programming assignments automatic grading: review of tools and implementations. In INTED, pages 5691-5700, 2013.

[4] F. P. Deek, K.-W. Ho, and H. Ramadhan. A critical analysis and evaluation of web-based environments for program development. The Internet and Higher Education, 3(4):223-269, 2000.

[5] F. P. Deek and J. A. McHugh. A survey and critical analysis of tools for learning programming. Computer Science Education, 8(2):130-178, 1998.

[6] C. Douce, D. Livingstone, and J. Orwell. Automatic test-based assessment of programming: A review. Journal on Educational Resources in Computing (JERIC), 5(3), 2005.

[7] M. Gómez-Albarrán. The Teaching and Learning of Programming: A Survey of Supporting Software Tools. The Computer Journal, 48(2):130-144, 2005.
[8] P. Gross and K. Powers. Evaluating assessments of novice programming environments. In ICER, pages 99-110, 2005.

[9] M. Guzdial. Programming environments for novices. In Computer Science Education Research, pages 127-154. 2004.

[10] P. Ihantola, T. Ahoniemi, V. Karavirta, and O. Seppälä. Review of recent systems for automatic assessment of programming assignments. In Koli Calling, pages 86-93, 2010.

[11] C. Kelleher and R. Pausch. Lowering the barriers to programming: A taxonomy of programming environments and languages for novice programmers. ACM Computing Surveys, 37(2):83-137, 2005.

[12] H. Keuning, J. Jeuring, and B. Heeren. Towards a systematic review of automated feedback generation for programming exercises - extended version. Technical Report UU-CS-2016-001, 2016.

[13] N.-T. Le and N. Pinkwart. Towards a classification for programming exercises. In Workshop on AI-supported Education for Computer Science, pages 51-60, 2014.

[14] N.-T. Le, S. Strickroth, S. Gross, and N. Pinkwart. A review of ai-supported tutoring approaches for learning programming. In Advanced Computational Methods for Knowledge Engineering, pages 267-279. 2013.

[15] D. C. Merrill, B. J. Reiser, M. Ranney, and J. G. Trafton. Effective tutoring techniques: A comparison of human tutors and intelligent tutoring systems. Journal of the Learning Sciences, 2(3):277-305, 1992.

[16] S. Narciss. Feedback strategies for interactive learning tasks. Handbook of research on educational communications and technology, pages 125-144, 2008.

[17] J. C. Nesbit, L. Liu, Q. Liu, and O. O. Adesope. Work in Progress: Intelligent Tutoring Systems in Computer Science and Software Engineering. In ASEE Annual Conference $\mathcal{E}$ Exposition, pages 1-12, 2015.

[18] A. Pears, S. Seidman, L. Malmi, L. Mannila, E. Adams, J. Bennedsen, M. Devlin, and J. Paterson. A survey of literature on the teaching of introductory programming. SIGCSE Bull., 39(4):204-223, 2007.

[19] N. Pillay. Developing intelligent programming tutors for novice programmers. SIGCSE Bull., 35(2):78-82, 2003.

[20] K. A. Rahman and M. J. Nordin. A review on the static analysis approach in the automated programming assessment systems. In National conference on programming, 2007.

[21] R. Romli, S. Sulaiman, and K. Z. Zamli. Automatic programming assessment and test data generation: a review on its approaches. In Int. Symp. in Information Technology, pages 1186-1192, 2010.

[22] V. J. Shute. Focus on formative feedback. Review of Educational Research, 78(1):153-189, 2008.

[23] M. Striewe and M. Goedicke. A review of static analysis approaches for programming exercises. In Computer Assisted Assessment. Research into E-Assessment, pages 100-113. 2014.

[24] M. Ulloa. Teaching and learning computer programming: a survey of student problems, teaching methods, and automated instructional tools. SIGCSE Bull., 12(2):48-64, 1980. 